Synthetic biology and the search for alternative genetic systems : Taking how-possibly models seriously

Koskinen, Rami Santeri

2017-05-05

Koskinen , R S 2017 , ' Synthetic biology and the search for alternative genetic systems :

Taking how-possibly models seriously ', European Journal for Philosophy of Science, vol. 7

pÿ, no. 3 , pp. 493506 . https://doi.org/10.1007/s13194-017-0176-2

http://hdl.handle.net/10138/228525

https://doi.org/10.1007/s13194-017-0176-2

acceptedVersion

Downloaded from Helda, University of Helsinki institutional repository.

This is an electronic reprint of the original article.

This reprint may differ from the original in pagination and typographic detail.

Please cite the original version. 


\title{
Synthetic biology and the search for alternative genetic systems: Taking how- possibly models seriously
}

\author{
Rami Koskinen, University of Helsinki
}

\begin{abstract}
Many scientific models in biology are how-possibly models. These models depict things as they could be, but do not necessarily capture actual states of affairs in the biological world. In contemporary philosophy of science, it is customary to treat how-possibly models as second-rate theoretical tools. Although possibly important in the early stages of theorizing, they do not constitute the main aim of modelling, namely, to discover the actual mechanism responsible for the phenomenon under study. In the paper it is argued that this prevailing picture does not do justice to the synthetic strategy that is commonly used in biological engineering. In synthetic biology, howpossibly models are not simply speculations or eliminable scaffolds towards a single how-actually model, but indispensable design hypotheses for a field whose ultimate goal is to build novel biological systems. The paper explicates this by providing an example from the study of alternative genetic systems by synthetic biologist Steven Benner and his group. The case will also highlight how the method of synthesis, even when it fails, provides an effective way to limit the space of possible models for biological systems.
\end{abstract}

\section{Introduction}

Many scientific models in biology can be regarded as how-possibly models. These depict things as they could be, but do not necessarily capture actual states of affairs in the real world. Examples range from early characterizations of the electro-physiological underpinnings of the action potential in neuroscience to simulations of hypothetical predator-prey systems in ecology. In their usual 
application, how-possibly models are identified with how-possibly explanations. ${ }^{1}$ According to Resnik (1991: 144), "[s]cientists from nearly every biological field give how-possibly explanations of the phenomena they study." Ever since Darwin, evolutionary biologists have given how-possibly explanations for biological adaptations based on natural selection. When molecular biologists are sketching alternative mechanisms for a newly found phenomenon, they are also working with howpossibly models in this sense. These models may lack full empirical support of a finished explanation, but are nevertheless supposed to provide some kind of scientific understanding.

In contemporary philosophy of science, how-possibly models are often treated as second-rate explanations or theoretical tools (e.g., Rosenberg 2006: 45; see also Craver 2006: 361, 2007: 112; Resnik 1991: 146). Many of them can be regarded as "speculations" (Resnik 1991: 144-145), or mere "just-so stories" (Forber 2010: 36). How-possibly models are sometimes conceptualized as vague schemas that arise from abstract mathematical modeling that is insensitive to biological details (Craver and Darden 2013: 34). They may be important heuristic tools in the early stages of theorizing (Resnik 1991; Reydon 2012), but do not constitute modelling's main aim, namely, to discover the actual mechanism responsible for the phenomenon under study.

In the present paper I will argue that this current view concerning the role of how-possibly models is too narrow. It may be a good approximation in the context of scientific analysis of natural systems where research advances through the methods of decomposition and localization (Bechtel and Richardson 2010). It is often the case that scientists are interested in understanding some welldefined actual target system. However, it is also true that often the target of investigation is a more abstract feature of the living world that might require studying objects or phenomena that do not exist, at least at the moment of investigation (Dawkins 1986: 73; Dennett 1995: Ch. 5). Instead of inquiring into how things actually are, it is natural to interpret these kinds of studies as focusing on how things possibly could be. Furthermore, I hold that this is especially true in the context of the synthetic strategy that is commonly used in biological engineering.

\footnotetext{
${ }^{1}$ The term "how-possibly explanation" goes back to Dray (1957), who wanted to provide a philosophical account of certain kinds of explanations in the historical sciences. The following presentation does not draw much on the details of Dray's original account. See Reydon (2012) on how Dray figures in contemporary approaches to how-possibly explanations in the philosophy of biology.
} 
In the new field of synthetic biology, researchers use how-possibly models to study what may be called potential biological systems. I argue that in the hands of bioengineers, how-possibly models are not just speculations or eliminable scaffolds towards one how-actually model, but rather design hypotheses for a field whose ultimate goal is to build novel biological systems and "re-wire" existing ones. Apart from their more traditional explanatory purpose, how-possibly models can function as ways of studying, and ultimately concretizing, biological possibilia.

I explicate this role further by providing an example from the study of alternative genetic systems by synthetic biologist Steven Benner and his group. These are artificially constructed chemical systems that differ structurally from natural DNA, but which are nevertheless purported to have the same ability to carry genetic information. Because these systems do not exist in nature, biologists have thus far been only able to speculate about the possibility of these systems. At their heart, these hypotheses are based on the possibility of having alterations to the classic Watson-Crick model of genetic material. However, synthetic biologists are now in a position to study the feasibility of these systems in concrete terms. The case study is specially selected to also highlight how the synthetic method, even when it fails, provides an effective way to limit the space of possible models for biological systems. This has effects on the study of potential and actual natural systems alike.

The outline of the paper is as follows. In Section 2, I begin by providing a philosophical background for theorizing about how-possibly models. I will show how different contrasts for howpossibly explanations and models that have been prominent in the philosophy of science literature (namely, why-necessary and how-actually) have effects on how we are to understand their role in synthetic biology research. In Section 3, I develop and explicate these ideas with a case study on the engineering of alternative genetic systems. Section 4 concludes by drawing some further implications for the study of the relative contingency of biological systems.

\section{From actual to potential biological systems}

In his influential account of modern "Darwinian reductionism" in biology, Alexander Rosenberg contrasts how-possibly explanations with why-necessary explanations. ${ }^{2}$ For Rosenberg, biologists should always opt for the latter type. According to him (2006: 43-44.):

\footnotetext{
${ }^{2}$ Here Rosenberg seems to follow Dray.
} 
[T]here is an important asymmetry between how-possible and why-necessary explanations that philosophers of history recognized. Once a how-possible explanation has been given, it makes perfect sense to go on and ask for a why-necessary explanation. But the reverse is not the case. Once a why-necessary explanation has been given, there is no point asking for a how-possible explanation.

I will not delve into the details of Rosenberg's view here. I will just note that it is at least prima facie easy to see how a why-necessary explanation for a certain phenomenon radically deflates the value of a how-possibly explanation for that same phenomenon. Although it might be pragmatically very difficult to acquire why-necessary explanations, once they are given there is little or no room left for wondering whether alternative explanations could do the same job. In this picture, howpossibly explanations and their accompanying how-possibly models are second-rate theoretical tools. However, contrasting how-possibly explanations with why-necessary ones is not the only way to draw the contrast. In contemporary philosophy of science, how-possibly explanations are most often contrasted with how-actually explanations.

According to the most influential contemporary account of explanation in the philosophy of biology, namely, that of mechanistic explanation, the aim of the life sciences is seen as the discovering and modelling of mechanisms that "produce, underlie, or maintain a phenomenon" that is being studied (Craver and Darden 2013: 15). The mechanistic strategy of modelling is typically conceptualized as proceeding by constraining a space of possible mechanisms for a given phenomenon or function. According to Craver (2007: 31), the space of possible mechanisms contains all the mechanisms that could possibly explain a phenomenon. By explicating a particular point in this space, scientists construct a how-possibly explanation or model. Furthermore, it is often assumed that there is one true or correct mechanism, the details of which are ideally captured in a finished how-actually model. Between these two extremes are intermediate how-plausibly models, which form a more tightly constrained subset of how-possibly models, but still lack the full empirical support of a how-actually model (Craver and Darden 2013: 34-35).

According to the mechanistic picture of biological modelling strategy, a successful search for explanation should ideally converge on a single how-actually model, and divergent how-possibly models that differ in their mechanistic details should be discarded as superfluous and scientifically incorrect. According to Craver (2007: 131), “[d]istinguishing good explanations from bad requires that one distinguishes real components from fictional posits. The most dramatic examples of fictional posits include animal spirits, entelechies, and souls, but fictitious entities can be far more mundane than these." He concludes that many how-possibly models "require parts (and activities) 
that do not exist" (Craver 2007: 131, my emphasis). Because scientists want their models to work and, in particular, do not want to commit themselves to any kind of spooky nonexistent entities, how-possibly models are considered as something that should be eliminated when conducting serious research. As in the case of Rosenberg's aspiration for why-necessary explanations, the mechanists' search for how-actually models also seems to considerably deflate the scientific status of how-possibly explanations and models.

However, what I will argue is that unlike in the case where researchers are in a possession of a whynecessary explanation, given a how-actually explanation or model there is still place for studying how-possibly models that is scientifically valuable. In particular, one can still legitimately ask: Why this particular system design and not some other possible one? Indeed, I am going to make the even stronger suggestion that apart from their more traditional explanatory purport, how-possibly models can maintain a function as tools for studying biological possibilia.

The mechanistic analysis of modelling strategy is surely a good approximation within the context of scientific analysis of natural systems where research advances through the methods of decomposition and localization (Bechtel and Richardson 2010). The idea of starting from a range of possible models and then working towards one how-actually model seems to make much sense when one considers the general purpose of biological investigation. For example, given that one of the main aims of science is to provide empirically adequate generalizations that unify phenomena as much as possible, focusing on how things actually work is a neat idea and surely a good starting point. Another reason that is especially prominent in the life sciences is the ability to effectively intervene on various target systems for medical purposes (see Craver 2007). Why would scientists bother wasting their time with mere how-possibly models that do not provide good access to actual phenomena, not to mention ways to effectively intervene on them?

However, the characterization of actual states of affairs does not exhaust all the goals of biological investigation. It is often said that an important thing about mechanistic understanding is the ability to answer "what-if-things-had-been-different" questions (e.g., Craver 2006: 358, following Woodward 2003). This is certainly true in the sense that, ideally, when a mechanism is fully understood (i.e., our best model of it contains no black boxes left to open), we are able to reliably predict its output for a range of input and parameter values and even manipulate its functioning. Knowing how an actual system operates as accurately as possible gives us more effective ways to handle typical counterfactual questions that arise in science (cf. Craver 2006). But this kind of access to the full mechanistic details of actual target systems forms only one part of contrafactual reasoning that is of interest to scientists. Sometimes, especially when dealing with more theoretical 
issues, scientists who ask "what-if-things-had-been-different" questions are not in fact inquiring how accurately we understand the parts and workings of some actual mechanism. Rather, I suggest that they might be wondering whether the mechanism (or the system in general) itself could be —or could have been - different. It is in this way that, instead of being just an eliminable scaffold on the way towards a how-actually model, a how-possibly model can become the main object of inquiry in its own right.

Taking how-possibly models for biological systems seriously in the above sense might mean two things. Firstly, it might simply mean taking seriously the general strategy of "turning the tables around", that is, focusing research on what is possible instead of actual in the biological world. For example, Grüne-Yanoff (2013: 858) briefly mentions the idea that some models in evolutionary biology might be used to study the possible capabilities of non-actual biological systems. This is akin to an exploration of uncharted territory where rather few things limit the search space. Secondly, and more importantly for the purposes of this paper, it might mean that one is committed to the study of some particular how-possibly model or set of models for a phenomenon for which there already is a well-confirmed how-actually model. The second version has the nice advantage of already being existential proof of the phenomenon or function being indeed realizable. We can then investigate whether it can be achieved by means of some alternative mechanism; the strategy is essentially contrastive in nature. ${ }^{3}$ Indeed, this is something that is done in many quarters of biological engineering and particularly in the field of synthetic biology.

Synthetic biology is a nascent field of biological engineering that has become ever more visible during the past decade. Its purpose is to build novel, predictably behaving, and functionally sound biological systems and devices with engineering methods (see Elowitz and Lim 2010; Endy 2005). Its products have been expected to provide great benefits to medicine, sustainable agriculture, and energy industry among other things. The field is characterized by a particularly strong interdisciplinary setup. Indeed, many of its proponents are from disciplines outside the traditional biological sciences. Some have their background in applied sciences like electrical engineering and computer science. Others are from more orthodox fields like physics and mathematics. The theoretical roots of synthetic biology are in the technical advancements of molecular biology

\footnotetext{
${ }^{3}$ On the importance of this kind of reasoning in evolutionary biology, where actuality is contrasted with nonexistent but nearby possibilities or alternatives that occupy the same "design space", see Dennet (1995: 102-103, and the subsequent Ch. 5) and Green (2015: 633).
} 
research of the past few decades and also in the more recent systems biology approach (Cameron, Bashor, and Collins 2014).

As in the case of more traditional life science research, mechanistic understanding and modelling of biological systems is at the heart of synthetic biology. In a sense, synthetic biology can be seen as taking them even further. As their methodological starting point, synthetic biologists approach biological systems as if they were technical artifacts susceptible to engineering design. Because this requires an excessive ability to manipulate existing biological systems, synthetic biology is often portrayed as the ultimate test for our mechanistic understanding of the living world in general (Endy 2005). ${ }^{4}$ However, at the same time these bioengineers are also expanding biological understanding over and above naturally occurring systems (Elowitz and Lim 2010; Morange 2009; Ruiz-Mirazo and Moreno 2013). Some of the synthetic systems that have been built, for instance artificial genetic circuits, can be seen both as new biological objects in their own right and as certain types of concrete, but theoretical models of what kind of potential design principles are biologically feasible (Knuuttila and Loettgers 2013). Although the study of these potential biological systems is targeted at certain very specific types of how-possibly models for biological systems, they can be seen as enriching our understanding of biology in terms that go beyond mere acts of tinkering.

For example, it has been suggested that synthetic design methods might be able to provide valuable information about the nature and limits of the evolution of gene regulation:

[An important problem in evolutionary biology is] why the genetic network architectures we observe in Nature have evolved to solve a particular problem an organism faces in its environment. This challenge is often complemented by the question of which selective forces (i.e. environmental or cellular conditions) have shaped the biological systems we observe in modern organisms. The null hypothesis is simply that a particular architecture has arisen by non-selective forces and that multiple architectures would be sufficient to achieve the biological functionality observed. (Bayer 2010: R775.)

In normal evolutionary research, these kinds of questions are often difficult to evaluate because the relevant sample might be biased by evolutionary contingency (Beatty 1995; Raerinne 2015). Many specific functions are found only in a very limited number of systems or model organisms; or, at the other extreme, the function or trait in question can be almost universal, making it impossible to

\footnotetext{
${ }^{4}$ Craver and Darden (2013: 92-94) also mention the importance of engineering or "build it test" as an effective way to further refine scientific understanding of biological mechanisms.
} 
draw any inferences regarding the relevant selective pressures. As Sterelny and Griffiths (1999: 232) put it: "There is a striking fact about life — surprising limitations on the range of the possibleabout which natural selection is silent." Modeling the mechanistic details of the actual system in ever greater detail does not seem to be of much help here. However, thanks to synthetic biology and other forms of biological engineering, evolutionary studies are no longer necessarily restricted by the availability of naturally occurring systems: "The construction of synthetic versions of natural circuits is a powerful way to interrogate questions of 'why' in biology" (Bayer 2010: R775). Endy also defines one of the main goals of synthetic biology as follows: "[...] synthetic biology provides an opportunity to test the hypothesis that the genomes encoding natural biological systems can be 're-written', producing engineered surrogates that might usefully supplant some natural biological systems" (Endy 2005: 449). Besides providing potentially valuable applications, artificial synthetic systems can provide insight into whether it is possibly to build functional biological systems that utilize designs different from those found in nature (Sprinzak and Elowitz 2005).

The re-design strategy depicted here limits its focus to systems that differ in their underlying architecture, but that are nevertheless capable of realizing the same higher-level function. It is reminiscent of the situation that philosophers call multiple realizability. As Weiskopf (2011: 243) suggests, the strategy of studying different how-possibly models for a common target phenomenon requires taking at least the possibility of multiple realizability seriously. Because how-possibly models are often presented in exactly this kind of situation where they are in a sense explanatory rivals - function-equivalent alternatives - for one and the same phenomenon, it is easy to see how they fit into the conceptual scheme of "biological rewriters". One of the most compelling examples of this kind of research comes from the study of artificial genetic systems that can be regarded as functional alternatives to our natural DNA. It is there that how-possibly models, on top of their more traditional explanatory purport, have the role of explicit and contrasting design hypotheses.

\section{Alternative alphabets for life's code}

Why is the genetic code based on the DNA molecule? Is it a functional necessity, or just a historical accident? The philosopher of science Stuart Glennan (2010: 372) states:

[I]t certainly does not follow [...] that DNA is the only possible realizer of the gene function within the actual world. We know that RNA carries genetic information in some viruses. But beyond this, it is likely that in a world with the same physical laws, there could be a wide variety of molecules and other mechanisms that could code and replicate information in the 
way that our DNA-based genes do. Indeed, if we were to find life in distant parts of the universe we would be exceedingly surprised if the mechanisms of heredity in these life forms would closely resemble ours. Close similarity would be evidence for common descent.

According to Glennan, it is a common mistake of philosophers to think that basic laws of nature somehow "fix" the realizations of functional entities. Rather, functional properties are produced by complex mechanisms, and multiple different mechanisms can often implement a particular function (Glennan 2010: 371-372). What Glennan seems to imply here is that people tend to easily mix how-actually accounts with why-necessary ones. As I have argued, this is simply not warranted. However, although actuality does not imply necessity, it does not always rule it out either. Because the sample size of life on Earth is one, there is no straightforward empirical way to investigate this issue.

In his famous booklet What is Life? the physicist Erwin Schrödinger (1944) originally proposed an inspirational how-possibly model for genetic material in which genes are hypothesized as consisting of some kind of aperiodic crystals. This was nine years before Watson and Crick's discovery of the structure of the DNA molecule. Because of their groundbreaking work, we, of course, now have an excellent how-actually model for the implementation of the genetic material. However, as successful as their model has turned out to be, it does not really answer all the why-questions that can be raised regarding the material nature of the genetic code. To answer these questions would require contrasting DNA with some other plausible how-possibly models for genetic material and hoping for some principled clue as to why nature has opted for this particular solution.

Synthetic biologist and chemist Steven Benner has since the 1980's been studying what can be called alternative genetic systems (Switzer, Moroney, and Benner 1989). These are artificial chemical structures that are supposed to have the essential functional features of a genetic system, but that are nevertheless different from the structural design of familiar DNA and RNA molecules. According to Benner:

In a version popular today in some engineering communities, [synthetic biology] seeks to use natural parts of biological systems (such as DNA fragments and protein "biobricks") to create assemblies that do things that are not done by natural biology (such as digital computation or manufacture of a speciality chemical). [...] Among chemists, "synthetic biology" means the opposite. Chemist's "synthetic biology" seeks to use unnatural molecular parts to do things that are done by natural biology. Chemists believe that if they can reproduce biological behavior without making an exact molecular replica of a natural living system, then they have 
demonstrated an understanding of the intimate connection between molecular structure and biological behavior. If taken to its limit, this synthesis would provide a chemical understanding of life. (Benner, Yang, and Chen 2011: 372; emphasis in original.) $)^{5}$

For the last 25 years Benner has studied a wide range of different chemical systems that could potentially be used to fulfill the same role as DNA/RNA in naturally evolved organisms. Groups like his have managed to construct many interesting variants that have at least some of the features required of a functional genetic system, and new exciting results are frequently being reported from scientists working at the junction of synthetic biology and chemistry (e.g., Malyshev at al. 2014; Marlière et al. 2011; Thyer and Ellefson 2014).

Although these studies are still not conclusive, they nevertheless provide good reasons to believe that alternative genetic systems are a live possibility. To make the continued hegemony of DNA as the biochemical medium of choice for genetic material seem even less secure, Benner noted in an interview published in Nature in November 2012 that " $[t]$ he first thing you realize is that [DNA] is a stupid design," further insisting that "[i]f you were a chemist setting out to design this thing, you would not do it this way at all" (Kwok 2012: 516). For example, the fact that important structural features of DNA are implemented by hydrogen bonding seems unnecessarily daring for Benner. Because of basic chemical laws, hydrogen bonds are easily broken up in water-something that living cells are full of (Kwok 2012: 516).

However, it is one thing to criticize the structural design of DNA, and another to show that any other chemical solution would be able to perform the same functions. Because all known organisms have their genetic code based on DNA/RNA, the possibility of "alternative genetic alphabets" requires strong empirical proof (Thyer and Ellefson 2014: 291). One would expect there to be good chemical and evolutionary reasons for DNA to be the medium for genetic information. Although it is nowadays recognized that biological solutions are not always optimal in the strong sense, they are nevertheless often extremely robust and efficient (see, e.g., Wagner 2005). Because so much

\footnotetext{
5 The version of synthetic biology that Benner advocates here (“chemists' version") suits well the idea of how-possibly models as alternative design hypotheses because of the built-in contrastiveness, or shared functionality, of the engineered systems. The idea could also be applied to those branches of synthetic biology that try to engineer completely new functions ("things that are not done by natural biology"), but this would require stretching the notion of a how-possibly model somewhat. It is also beyond the scope of this article.
} 
complex, evolutionarily successful life is based, at the bottom level, on the structural features of DNA, it simply cannot be that inadequate as a molecule. However, it is also because of this most intense of dependencies that it is actually very difficult to make many far reaching biological conclusions about the nature of DNA. It is a deeply generatively entrenched feature of the living world; other things both crucially depend on it and at the same time tend to further stabilize its role (Wimsatt 2007: 135-136).

To understand the requirements for an adequate genetic system, I will first have to examine the definition of a living system that Benner and others advocate. This is a working definition, which means that it is open for revision. It nevertheless captures many of the features that biologists take to be essential for living systems. In his work, Benner follows the so-called "NASA definition" of life as a self-sustaining chemical system capable of Darwinian evolution (Benner, Yang, and Chen 2011: 375). Although this definition leaves many facts for further refinement, it nevertheless already makes some empirical bets by, for example, ruling out genuinely Lamarckian systems. As Benner himself notes, we have no reasons to believe that even Lamarckian systems would be strictly impossible (Benner, Yang, and Chen 2011: 375). However, we have to start from somewhere. Moreover, Darwinism has obviously proven to be a very successful evolutionary framework. In a sense, synthetic biologists can help to take the refinement of this theory even further by trying to come up with some unfamiliar chemical systems that can be subsumed under it. Benner's abstract model of a genetic system has three features: (1) the ability to carry biological information, (2) the ability to transmit biological information, and (3) the ability to support Darwinian evolution (based on Benner, Yang, and Chen 2011). In practice, the above list can be thought to also encompass some implicit auxiliary assumptions à la Duhem and Quine. Examples of these could be some kind of linear arrangement of the code, or the overall chemical and thermodynamic stability of its structure (see Szathmáry 2003). These general features can also be broken down into smaller mechanisms or causal role functions that make them physically feasible. For example, the encoding of biological information is often taken to require some kind of chemical specificity, like bonding, lock-and-key complementarity, and so on. This brings the whole enterprise closer to the how-plausibly end of all conceivable possibilities.

Although the above list seems quite simple, it contains an implicit tension that makes it more difficult to achieve all of the requirements simultaneously. It is obvious that without the first requirement, we would simply have no code at all - genetic or other kind. However, the mere ability to store information is not that interesting a property in itself. The code must also be able to transmit information from one system to another. It is only after this step is fulfilled that we can 
actually speak about inheritance. Taken in isolation, requirements (1) and (2) suggest that the more accurate the functions in question are, the better the medium is in realizing the code. In a sense this is true. A system that transmits its information content so poorly that descendant systems hardly resemble their parent systems would clearly not be able to support Darwinian life. However, what requirement (3) implicitly insists is that although the copying process should be reliable, it should not be completely certain. Otherwise no variation is ever going to accumulate, and the system can only produce an endless army of genetically identical clones. Again, the space of possible models for genetic material is in this way constrained before any considerations of the physical medium has taken place.

According to Benner, the Watson-Crick model of natural DNA suggests many possible alternative designs that could potentially implement genetic information. At first these were based on various cartoon models that fed on simple permutations of different molecules with the familiar size and bonding complementarities of DNA (Benner et al. 2016). However, the experimental work had to start from somewhere. Many synthetic biologists' original expectation was that the best place to begin changing the chemical basis of the genetic code was the sugar backbone of natural DNA molecules. This is because the informational specificity of the genetic code is often thought to lie in the highly specific complementary base pairings between the nucleobases adenine-thymine (A-T) and cytosine-guanine $(\mathrm{C}-\mathrm{G})$. The backbone was believed to be just a contingent structure, a kind of molecular "scaffold", whose purpose is to support the real sources of information (Benner, Yang, and Chen 2011: 376). However, as it turned out after numerous trials, Benner and his team were unable to achieve functionally stable molecules by changing the sugar backbone. For example, backbones made of glycerol units turned out be too flexible and the whole structure broke down in normal temperatures; the nucleobase bindings alone were not strong enough to hold the structure together (Benner, Yang, and Chen 2011: 377).

In addition to sugars, the DNA backbone features phosphates. Similar results were attained by Benner and his team when they tried to change the phosphates as in the case of the sugars. For example, when the phosphates were replaced by synthetic oligosulfones, the structure tended to fold onto itself (Benner, Yang, and Chen 2011: 378). This was bad news, because folded structures are not specific enough to ensure faithful pairing. Moreover, if the structure is stripped from its repeating charges that are manifest in the phosphates, it might hamper its mutability; remember that the ability to evolve is one of the functional requirements of life that Benner advocates in his working model (Benner, Yang, and Chen 2011: 379-380). Thus, the sugar and phosphate backbone 
with its repeating charges seemed to be a necessary feature of a biologically plausible genetic system capable of Darwinian evolution. ${ }^{6}$

This meant that in order to achieve the grand goal of an alternative genetic system, the changes must be made to the nucleobases. After trying out numerous working hypotheses or possible models for a genetic system that is based on alternative or "unnatural" alphabets, Benner and his team finally arrived at the six-letter alphabet (A, T, C, G, P, Z). Using methods like PCR, they were able to add the bases $\mathrm{P}$ and $\mathrm{Z}$ to a system based on the natural $(\mathrm{A}, \mathrm{T}, \mathrm{C}, \mathrm{G})$ alphabet. These bases were selected because bonding between them has experimentally been shown to be very strong and specific. Furthermore, unlike in the case of alternative backbones, the new bases can support Darwinian evolution: in the case of the (A, T, C, G, P, Z) alphabet, the new bases have been shown to be mutable to natural bases. Also, and perhaps even more interestingly, $\mathrm{C}$ and $\mathrm{G}$ could mutate to give $\mathrm{P}$ and $\mathrm{Z}$ (Benner, Yang and, Chen 2011: 384).

Benner's alphabet has some very interesting biological properties. First, it is a mix of both natural and artificial biochemical, or genetic, "letters". Second, its cardinality differs from that of the natural DNA/RNA alphabet. These two features make it possible to use the new alphabet to study both disparate and expanded genetic alphabets at the same time - a double benefit. These features also make it an interesting case study to study various how-possibly models of genetic systems.

Do the incorporated bases $\mathrm{P}$ and $\mathrm{Z}$ radically differ from the molecular structure of those of the natural nucleobases? This is somewhat debatable. It is true that the molecular mechanisms of pairing between them resemble those of $\mathrm{A}-\mathrm{T}$ and $\mathrm{C}-\mathrm{G}$. However, they are still structurally different molecules. It was certainly far from clear that these bases could be successfully inserted into a natural alphabet system. This is especially so because they tend to change the "dynamics" of the whole structure. The more different types of parts that can possibly interfere with each other there are, the more likely it is that the whole system will fail to be able to perform its functions properly; the basic parts of a mechanism often seriously constrain its space for possible models (cf. Craver and Darden 2013: 105). Also, the informational change that is brought by the new nucleobases can be as interesting as the change in structural features. With alphabets of different cardinality, it is possible to test the idea of whether the functions of living systems can be coded in ways that differ

\footnotetext{
${ }^{6}$ In the case of some xeno nucleic acids, or XNAs, researchers have been able to change the sugar backbone of DNA molecules. However, it is not clear whether these systems can support life. See Schmidt (2010).
} 
from the familiar four-letter system. While some previous models had been cautiously in favor of this (e.g., Szathmáry 2003), Benner's (A, T, C, G, P, Z) alphabet is one of the first experimental results to give compelling reasons to believe that they can.

Although it is still early days for these kinds of studies, they hint at the possibility that several different solutions for genetic systems are possible and materially realizable. In addition to Benner's group, many others have also been working on synthetic genetic systems and alternative alphabets. For example, Marlière et al. (2011) report a successful incorporation of a new nucleobase 5chlorouracil into a laboratory strain of E. coli, while Malyshev et al. (2014) produced similar experiments with the pair d5SICSTP-dNaMTP. What is remarkable is that both cases exemplified robust functionality with no obvious biological pitfalls. Successes like these make scientists more confident that similar results could be obtained in other related biological domains. From alternative and expanded alphabets is not a long road to systems that utilize different genetic codes or even utilize unnatural amino acids (Benner et al. 2016). The possibility of these kind of systems has already been a topic of modelling for some time in theoretical biology (see Szathmáry 2003; Wagner 2005: 18-21, 195-199). Although Benner is the first to admit that chemists are still far from being able to simply draw the molecules that, once synthesized, "will together have the properties that we value in living systems" (Benner et al. 2016), synthetic biologists are now in a position to take these models as abstract design templates and try to study their feasibility in concrete terms.

\section{Conclusions}

How-possibly models are dismissed in the philosophical literature because of their failure to represent actual states of affairs in the biological world. This is often justified, when the purpose of research is primarily to analyse the workings and composition of natural systems. However, this does not exhaust all the goals of biological investigation. I have argued that in new fields like synthetic biology, how-possibly models can have a novel and important function as design hypotheses - ways of studying, and ultimately concretizing, biological possibilia. These contingently non-existent systems can be seen as exploring potential biology that goes beyond actual evolutionary designs.

It is true that when synthetic biologists manage to successfully concretize a potential biological system, its accompanying how-possibly model loses its modal qualifier and instead becomes a howactually model. So, within the picture that I have sketched, how-possibly models do become 
discarded after all. Is this a problem for my argument? I do not think so. I have not been suggesting that there is any fundamental difference between how-possibly and how-actually models that, for example, allows them to track some kind of separate biological realities. Rather, what I have been trying to show is that the study of how-possibly models remains a viable research strategy even after the establishment of a successful how-actually model. Indeed, it is a strategy that is scientifically very fruitful.

In the absence of a why-necessary explanation, the possibility of alternative non-actual ways to realize a given system cannot be ruled out a priori. This is especially true in the context of the biological sciences, where evolutionary contingency often biases available designs. Biologists are rarely in a position to say that some feature of a living organism is necessarily the way it is, even if it is a good enough adaptation to a particular evolutionary problem in a particular environmental context. In fact, it can be argued that the only way to study the relative contingency of various biological systems is precisely to contrast actual systems with possible alternatives. I have shown how this is possible in at least some areas of biology by using methods from biological engineering.

Contrary to traditional how-actually-driven life-scientific practice, the how-possibly-driven engineering strategy of fields like synthetic biology can be used to explore the space of biological possibilities by taking established how-actually models for biological systems as a starting point, and then working towards realizing alternative and contrasting how-possibly models. If it succeeds, it opens up exciting new possibilities. For example, it might enable biologists to breed organisms that have access to larger sequence spaces, and that perhaps even utilize different amino acids and protein structures, than naturally evolved organisms (Schmidt 2010: 324). However, even if it does not, something valuable is still being learned. The failure to synthesize a particular system gives new information on what kinds of potential design principles are biologically feasible (Knuuttila and Loettgers 2013: 880) and in this way limits the space of possible models for biological systems. Furthermore, it can also shed new light on the nature of actual systems.

It might be that some of the structural features of DNA, like the repeating charges along its backbone, are essential: so-called forced moves in the space of available design (see Dennett 1995: 128-131). However, in the case of the familiar genetic alphabet, it seems that nature could have chosen otherwise, but for some reason it simply did not. It seems to be a partly contingent solution. Because it is no longer possible for evolution to change this - it would require overly drastic changes to the basic functional architecture of organismal design - the only plausible way to study these questions is to use synthetic design methods. This is also a good way to naturalize the notion 
of biological possibility: to show that how-possibly models and speculative scenarios in many fields of biology can be given a philosophically satisfying reading.

\section{References}

Bayer, T.S. 2010. Using synthetic biology to understand the evolution of gene expression. Current Biology 20: R772-R779.

Beatty, J. 1995. The evolutionary contingency thesis. In G. Wolters, J.G. Lennox, and P.

McLaughlin (eds), Concepts, theories, and rationality in the biological sciences. The second Pittsburgh-Konstanz colloquium in the philosophy of science. Pittsburgh: University of Pittsburgh Press, 45-81.

Bechtel, W., and R.C. Richardson. 2010. Discovering complexity: Decomposition and localization as strategies in scientific research (2nd ed.). Cambridge: The MIT Press.

Benner, S.A., Z. Yang, and F. Chen. 2011. Synthetic biology, tinkering biology, and artificial biology. What are we learning? Comptes Rendus Chimie 14: 372-387.

Benner, S.A., N.B. Karalkar, S. Hoshika, R. Laos, R.W. Shaw, M. Matsuura, D. Fajardo, and P. Moussatche. 2016. Alternative Watson-Crick synthetic genetic systems. Cold Spring Harbor Perspectives in Biology. doi: 10.1101/cshperspect.a023770

Cameron, D.E., C.J. Bashor, and J.J. Collins. 2014. A brief history of synthetic biology. Nature Reviews Microbiology 12: 381-390.

Craver, C.F. 2006. When mechanistic models explain. Synthese 153: 355-376. 2007. Explaining the brain: Mechanisms and the mosaic unity of neuroscience. Oxford: Oxford University Press.

Craver, C.F., and L. Darden. 2013. In search of mechanisms: Discoveries across the life sciences. Chicago: The University of Chicago Press.

Dawkins, R. 1986. The blind watchmaker: Why the evidence of evolution reveals a universe without design. Norton \& Company.

Dennett, D.C. 1995. Darwin's dangerous idea: Evolution and the meanings of life. New York:

Simon and Schuster.

Dray, W. 1957. Laws and explanation in history. Oxford: Clarendon Press.

Elowitz, M.B., and W.A. Lim. 2010. Build life to understand it. Nature 468: 889-890.

Endy, D. 2005. Foundation for engineering biology. Nature 438: 449-453.

Forber, P. 2010. Confirmation and explaining how-possible. Studies in History and Philosophy of Biological and Biomedical Sciences 41: 32-40. 
Glennan, S. 2010. Mechanisms, causes, and the layered model of the world. Philosophy and Phenomenological Research LXXXI: 362-381.

Green, S. 2015. Revisiting generality in biology: Systems biology and the quest for design principles. Biology and Philosophy 30: 629-652.

Grüne-Yanoff, T. 2013. Appraising models nonrepresentationally. Philosophy of Science 80: 850861.

Knuuttila, T., and A. Loettgers. 2013. Synthetic modeling and mechanistic account: Material recombination and beyond. Philosophy of Science 80: 874-885.

Kwok, R. 2012. DNA's new alphabet. Nature 491: 516-518.

Malyshev, A., K. Dhami, T. Lavergne, T. Chen, N. Dai, J.M. Foster, I.R. Corrêa Jr, and F.L. Romesberg. 2014. A semi-synthetic organism with an expanded genetic alphabet. Nature 509: $385-388$.

Marlière, P., J. Patrouix, V. Döring, P. Herdewijn, S. Tricot, S. Cruveiller, M. Bouzon, and R. Mutzel. 2011. Chemical evolution of a bacterial genome." Angewandte Chemie International Edition 50: 7109-7114.

Morange, M. 2009. Synthetic biology: A bridge between functional and evolutionary biology. Biological Theory 4: 368-377.

Raerinne, J. 2015. Evolutionary contingency, stability, and biological laws. Journal for General Philosophy of Science 46: 45-62.

Resnik, D.B. 1991. How-possibly explanations in biology. Acta Biotheoretica 39: 141-149.

Reydon, T.A.C. 2012. How-possibly explanations as genuine explanations and helpful heuristics: A comment on Forber. Studies in History and Philosophy of Biological and Biomedical Sciences 43: 302-310.

Rosenberg, A. 2006. Darwinian reductionism: Or, how to stop worrying and love molecular biology. Chicago: The University of Chicago Press.

Ruiz-Mirazo, K., and A. Moreno. 2013. Synthetic biology: Challenging life in order to grasp, use, or extend it. Biological Theory 8: 376-382.

Schmidt, M. 2010. Xenobiology: A new form of life as the ultimate biosafety tool. BioEssays 32: $322-331$.

Schrödinger, E. 1944. What is life? Cambridge: Cambridge University Press.

Sprinzak, D., and M.B. Elowitz. 2005. Reconstruction of genetic circuits. Nature 438: 443-448.

Sterelny, K, and P.E. Griffiths. 1999. Sex and death: An introduction to philosophy of biology. Chicago and London: The University of Chicago Press. 
Switzer, C., S.E. Moroney, and S.A. Benner. 1989. Enzymatic incorporation of a new base pair into DNA and RNA. Journal of the American Chemical Society 111: 8322-8323.

Szathmáry, E. 2003. Why are there four letters in the genetic alphabet? Nature Reviews Genetics 4: 995-1001.

Thyer, R., and J. Ellefson. 2014. New letters for life's alphabet. Nature 509: 291-292.

Wagner, A. 2005. Robustness and evolvability in living systems. Princeton, NJ: Princeton University Press.

Weiskopf, D.A. 2011. The functional unity of special science kinds. The British Journal for the Philosophy of Science 62: 233-258.

Wimsatt, W.C. 2007. Re-engineering philosophy for limited beings: Piecewise approximations to reality. Cambridge: Harvard University Press.

Woodward, J. 2003. Making things happen: A theory of causal explanation. Oxford: Oxford University Press. 